\title{
Magnetically Assisted Capsule Endoscopy for Endoscopic Examination of Esophagus and Stomach- Beginning of the End of Flexible Esophagogastroscopy!
}

\author{
Philip Daniel $^{1} \quad$ Surinder Singh Rana ${ }^{1}$ \\ ${ }^{1}$ Department of Gastroenterology, Postgraduate Institute of \\ Medical Education and Research (PGIMER), Chandigarh, India
}

\begin{abstract}
Address for correspondence Surinder S. Rana, MD, DM, FASGE, FSGEI, Department of Gastroenterology, Postgraduate Institute of Medical Education and Research (PGIMER), Chandigarh 160012, India (e-mail: drsurinderrana@gmail.com).
\end{abstract}

J Digest Endosc

Capsule endoscopy (CE) has gradually replaced radiological investigations as the procedure of choice for the evaluation of small bowel mucosal pathologies since its introduction in 2000s. Good patient tolerability and lack of need for sedation have led on to expansion of the indications of CE with attempts to use CE for the evaluation of other parts of the gastrointestinal tract like the upper gut and the colon. Observation of the esophagus and especially the stomach is difficult with conventional CE. The capsule usually transits the esophagus within seconds limiting adequate visualization of the esophageal mucosa. Also, the gastric lumen is larger in surface area and is much more difficult to be completely visualized on CE. While the antrum can be adequately evaluated, visualization of the body and fundus is suboptimal due to the larger surface area and inability to adequately distend the stomach due to lack of air insufflation. Inability to suck and remove liquid residue or bubbles and to perform diagnostic biopsies or other interventions have limited the use of conventional CE in the evaluation of gastric pathologies. For evaluation of antral vascular pathologies, CE has been proposed to be better than flexible gastroscopy as it visualizes the antrum under more physiological conditions without the effect of air insufflation that may compress ectatic vessels. ${ }^{1}$

To overcome the above limitations of $\mathrm{CE}$, investigators have tried new $\mathrm{CE}$ devices that have active locomotion systems. The movement of these devices can be controlled by the user from outside and the capsule can be maneuvered toward the area of interest. Two types of active locomotion systems have been developed with one having an intrinsic locomotion device in the capsule, whereas the second system relies on an external magnetic force to control the capsule movement. ${ }^{1,2}$ Tortora et al devised a CE system that had four motors to steer the capsule. In this procedure, the patient initially ingests $500 \mathrm{~mL}$ of clear liquid for adequate distension of the stomach. ${ }^{3}$ Thereafter, the capsule is ingested and the four propellers at the rear of the CE propel the capsule in the liquid filled stomach. The CE can be controlled wirelessly by the endoscopist using a joystick. This device has shown promise in in vitro and animal experiments. However, mechanical complexity as well as need for larger power supply to battery both the capsule and locomotion are important limitations of this system. ${ }^{2-4}$

In 2010, Keller et al used a capsule device (magnetic maneuverable capsule [MMC]; Given Imaging Ltd, Yoqneam, Israel; $31 \times 11 \mathrm{~mm}$ size, $7 \mathrm{~g}$ weight, battery life 10 hours, $256 \times 256$ pixels resolution, 4 frames/ second, 156-degree field of view) for examining the esophagus. This capsule device had magnetic disks placed inside one of its domes and could be controlled from outside the body using a handheld magnetic paddle. ${ }^{5}$ The authors demonstrated that the MMC could be longitudinally rotated and tilted along its axis for adequate mucosal inspection. Moreover, the MMC stayed longer in the esophageal lumen with better visualization of $\mathrm{Z}$ line as compared with the conventional esophageal capsule (ESO2, Given Imaging Ltd, Yoqneam, Israel). However, the magnetic force of the external paddle was not strong enough to hold the capsule at the GE junction and prevent its propulsion into the gastric lumen. Keller et al also evaluated the same device for the evaluation of stomach in 10 healthy volunteers and found that 7 subjects had good mucosal visibility where $>75 \%$ of the gastric mucosa was visualized, while the other 3 had moderate mucosal visibility. ${ }^{6}$

A CE system with similar principles, the MACE (magnetic-assisted capsule endoscope) system controlled by external magnetic paddles, was developed by Intromedic Company, Korea (MiroCam Navi, size $11 \times 24 \mathrm{~mm}$, weight $4.2 \mathrm{~g}$, battery life 8 hours, $320 \times 320$ pixels resolution, 3 frames/second, field of view 170 degrees). In a pilot study among 26 volunteers, Rehman et al demonstrated that the 
MACE system was able to provide adequate visualization of 88 to $100 \%$ of the upper gastrointestinal tract (esophagogastric junction $92 \%$, gastric cardia $88 \%$, fundus $96 \%$, body $100 \%$, incisura $96 \%$, antrum $96 \%$ and pylorus $100 \%$ ). They reported that the image quality was not so good at the esophagogastric junction and the pylorus due to rapid transit of the capsule across these parts. ${ }^{7}$ The same group used multiplanar reconstruction computed tomography modelling to determine the optimal sites for the placement of the capsule in the stomach that would lead to complete mucosal evaluation as well as the ideal site for the placement of the handheld magnetic paddle outside the body for optimal control of the capsule and reported encouraging results. ${ }^{8}$ MACE system has also been studied for the evaluation of upper gastrointestinal bleeding. ${ }^{9}$ MACE detected more focal lesions compared with conventional gastroduodenoscopy, but the number of significant lesions responsible for bleeding detected was similar in both the groups. Importantly, additional source of bleeding in the small bowel was detected by MACE in 18\%. Though visualization of most parts of the upper gastrointestinal tract was satisfactory, the authors reported suboptimal evaluation of some areas such as the esophagus, gastroesophageal junction, fundus, and the duodenal bulb. They also reported that the CE findings correctly predicted safe discharge for patients. This study suggested that MACE may have a role in the rapid evaluation of acute upper gastrointestinal bleeding and maybe more appropriate when the patient is not hemodynamically stable enough for a conventional diagnostic endoscopy.

The second type of magnetically controlled capsule is a device custom made for gastric evaluation, jointly developed by Olympus Medical Systems Corporation (Tokyo, Japan) and Siemens Healthcare (Erlangen, Germany) (size $31 \times 11 \mathrm{~mm}, 4$ frames/ second, 145 degrees field of view). The mobility of this capsule device named magnetically guided capsule endoscope could be controlled using low level magnetic fields ( 0.1 Tesla) delivered using magnetic resonance imaging equipment. In the pilot study published in 2010, Rey et al documented a technical success rate of $98 \%$. Adequate mucosal visualization reported was $75 \%$ in the cardia, $73 \%$ for fundus, $96 \%$ for body and $98 \%$ for the antrum of the stomach. ${ }^{1}$ This new capsule device was subsequently compared with the standard flexible gastroscopy in a nonrandomized blinded trial in 61 patients and $58.3 \%$ gastric lesions were picked up by both gastroscopy and CE. Fourteen lesions seen on gastroscopy were missed on CE and 31 lesions missed on gastroscopy were picked up by the $\mathrm{CE}$. The authors concluded that the overall diagnostic accuracy was similar for both the procedures. ${ }^{10}$ Another trial in 189 symptomatic patients showed that this CE had an accuracy of $90.5 \%$, sensitivity of $61.9 \%$, and specificity of $94.1 \%$ compared with conventional gastroscopy plus biopsy. ${ }^{11}$

The third type of magnetically controlled device developed used a robotic C-arm as the magnetic control (Ankon NaviCam, Ankon Optoelectronic Technology Co. Ltd, Wuhan, Shanghai, China; size $28 \times 12 \mathrm{~mm}$, weight $5.2 \mathrm{~g}$, battery life 8 to 10 hours, $480 \times 480$ pixels resolution, 2 frames/ second, field of view 140 degrees). The robotic C-arm greatly increased the accuracy of movement control compared with the handheld magnetic capsule endoscopes. ${ }^{12}$ In a pilot study published in 2012, 31 healthy volunteers underwent the procedure and > $75 \%$ of the gastric mucosa could be visualized in $79.4 \%$ of the subjects. Adequate visualization reported was $82.4 \%$ in gastric cardia, $85.3 \%$ in fundus, $100 \%$ in the body, incisura, antrum, and pylorus. Moreover, the maneuverability of the capsule device was reported to be good in $85.3 \%$ of the study subjects. ${ }^{12}$ Ching et al reported excellent visualization of all areas of the gastric mucosa using the Ankon NaviCam device. ${ }^{13}$ In a trial comparing this capsule device to conventional gastroscopy, the diagnostic accuracy was similar with an overall agreement of $91.2 \%$ among the two modalities. ${ }^{14}$ In a larger trial involving 350 patients with upper abdominal complaints, this device was reported to have a sensitivity of $90.4 \%$, specificity $94.7 \%$, positive predictive value $87.9 \%$, and negative predictive value $95.9 \%$ for picking up gastric lesions. Importantly, 95.7\% patients preferred this modality over a conventional gastroscopy. ${ }^{15} \mathrm{~A}$ recently published study used a newer second generation version of the NaviCam capsule and compared it to the older version. This capsule had a superior frame rate of eight frames/ second, better image resolution of $720 \times 720$ pixels, wider field of view of 150 degrees, more than 12 hours battery life and antijamming wireless technology for optimal control of the device. Evaluation of the esophageal mucosa and the Z-line was significantly better compared with the older device. Maneuverability and image quality were also superior and gastric transit time could be shortened with the newer version. However, lesion detection rates were similar between the two models. ${ }^{16}$

The best way to achieve adequate gastric preparation before performing $C E$ is a question that has not yet been sufficiently addressed. For adequate visualization, the stomach has to be properly distended, and obscuring secretions and bubbles have to be removed. Magnetically controlled CE works when there is a liquid interface enabling controlled movement of the capsule. Initially researchers attempted using gas forming powder to achieve adequate distension. However, the results were disappointing and now clear water is used to fill the stomach. Agents like simethicone are used for clearing bubbles and debris that could obstruct optimal visualization. ${ }^{17}$ Control of the capsule in the antrum against the propulsive peristaltic forces is another reason for concern. Some investigators have used antispasmodic drugs like scopolamine to reduce peristalsis but there is insufficient data regarding the benefit of the same. ${ }^{17}$

Magnetically controlled CE devices have also been studied for the assessment of esophageal diseases. Lien et al in a pilot study used a cable capsule endoscope that could be controlled using a handheld magnetic navigator to examine the upper gastrointestinal tract in 10 healthy volunteers. Esophageal transit speed was controlled using the cable attached to the capsule and completeness 
of the examination for all esophageal landmarks was $100 \% .{ }^{18}$ Chen et al demonstrated a novel technique for the examination of the esophagus by using a detachable string magnetically controlled capsule. Once the patient swallowed the capsule, it was allowed to pass till the gastric cardia. Then it was pulled back up using the attached string and the esophageal mucosa was examined. After completing the esophageal examination, the string was detached and the capsule was allowed to pass into the gastric lumen. ${ }^{19}$ A modification of the Ankon NaviCam capsule device with a detachable string has also been developed especially for esophageal examination. Compared with the standard wireless NaviCam device, this modified capsule gave better evaluation of the esophageal mucosa including the $Z$ line. Use of the string enabled repeated and adequate examination for any focal lesions. ${ }^{20}$

In a pilot study published recently in Gastrointestinal Endoscopy Journal, Beg et al used the wireless MiroCam Navi MACE system to evaluate utility of this device in diagnosing esophageal varices (EV) and Barrett's esophagus (BE). ${ }^{21}$ Fifty participants were included, including 17 with known EV, 17 with known BE, and 16 controls. These participants underwent the MACE procedure, with the operator blinded to previous endoscopy reports as well as indication for the procedure. All participants ingested $1 \mathrm{~L}$ water with added Simethicone prior to the CE procedure. The capsule was swallowed in the supine position with the head raised at an angle of 10 degrees and this prevented the rapid transit of the capsule through the esophagus due to gravity. Moreover, the investigators used the handheld magnetic paddle to control the passage of the capsule through the esophageal lumen and attempts were made to keep the capsule in the lumen as long as possible. Once this procedure was complete, all participants underwent esophagogastroduodenoscopy (EGD) where the endoscopist was blinded to the findings of the capsule study. MACE was able to diagnose EV with a sensitivity of $73.3 \%$ and specificity $100 \%$, whereas BE was diagnosed with sensitivity of $93.8 \%$ and specificity of $100 \%$. The investigators were able to hold the capsule within the esophageal lumen using the handheld magnet in 68\% patients. The mean esophageal transit time was $190 \mathrm{sec}-$ onds but with a wide range from 5 seconds to 634 seconds. Visualization of esophageal landmarks was comparable to EGD, but EGD scored better for the evaluation of the $Z$ line. Technical success was $94 \%$ with MACE compared with $100 \%$ with EGD and the patient comfort and tolerability were significantly better for the MACE procedure. This pilot study reported a reasonable accuracy for the detection of esophageal lesions for magnetically controlled CE with very high levels of patient comfort and acceptance. The EV detection rates were comparable, while the BE detection rates were better with the MACE system compared with dedicated esophageal capsules used in previous studies. Moreover, magnetically controlled CE has an advantage of adequate visualization of the gastric mucosa also. The authors concluded that with improvements in capsule technology, the ability to hold the capsule in the esophageal lumen and to modulate esophageal transit times may come under the full control of the endoscopist in the future.

\section{Commentary}

The advantage of magnetically controlled CE over conventional EGD is its noninvasive nature making it widely acceptable for patients. Unlike conventional EGD sedation is not required and patient tolerability has been excellent in most of the studies. Moreover, only minor adverse events like abdominal discomfort, nausea, and vomiting have been reported. Thus, this procedure may be safely used in those patients who are at high risk of undergoing a conventional diagnostic endoscopy. Capsule retention rates are expected to be similar to conventional CEs or may be even less as these devices will be predominantly used in those having suspected upper gastrointestinal tract pathologies.

Despite these advances, the CE science currently is still not perfect and further developments are needed before these magnetic CE can replace conventional flexible endoscopy. Esophageal transit times are highly variable even with magnetically controlled CE devices and proper visualization of gastric fundus is still a challenge. This challenge arises because of increased distance of fundal lumen to the skin surface compared with shorter distance between the antral lumen to the skin surface, making magnetic control of the capsule in the fundus difficult. ${ }^{8}$ Also, the fundus that is physiologically in a collapsed state needs to be distended adequately for optimal evaluation of the mucosal surface. Capsule devices incorporating air insufflation capability have also been developed and are in the experimental stage. Investigators have also tried to shorten the gastric transit time by trying to propel the capsule across the pylorus into the duodenum using magnetic force. Studies have shown inconsistent results, and as of now this capability is also suboptimal. ${ }^{22}$ Capsules with the potential of achieving therapeutic roles like biopsy sampling, hemoclip application, and thermal coagulation are also under development and will challenge flexible endoscopy in future. ${ }^{23,24}$ Incorporation of artificial intelligence with capsule endoscopic image interpretation is also undergoing clinical trials and artificial intelligence systems employing deep learning have shown positive results. ${ }^{25}$

It seems we are indeed entering an era of science fiction. In near future, magnetically control CE will enable patients to perform self-capsule endoscopic evaluation as part of gastric or esophageal disease/cancer screening programs. Only those capsule studies where an abnormality is detected by the artificial intelligence interface will need further physician guided evaluation and if needed gastroscopy for further evaluation and tissue sampling. ${ }^{26}$ High equipment cost is a limiting factor currently, which, however, is expected to come down once use of these devices becomes more widespread. Thus, there is a potential for CE to replace conventional endoscopy at least as a screening modality for use in large sections of the community for cancer screening programs. Advances in CE science may 
very well lead to CEs becoming the procedure of choice for both diagnostic and therapeutic endoscopies in the future.

\section{Conflict of Interest}

No conflicts of interest and no financial disclosures.

\section{References}

1 Rey JF, Ogata $\mathrm{H}$, Hosoe $\mathrm{N}$, et al. Feasibility of stomach exploration with a guided capsule endoscope. Endoscopy 2010;42(7):541-545

2 De Falco I, Tortora G, Dario P, Menciassi A. An integrated system for wireless capsule endoscopy in a liquid-distended stomach. IEEE Trans Biomed Eng 2014;61(3):794-804

3 Tortora G, Valdastri P, Susilo E, et al. Propeller-based wireless device for active capsular endoscopy in the gastric district. Minim Invasive Ther Allied Technol 2009;18(5):280-290

4 Carta R, Tortora G, Thoné J, et al. Wireless powering for a self-propelled and steerable endoscopic capsule for stomach inspection. Biosens Bioelectron 2009;25(4):845-851

5 Keller J, Fibbe C, Volke F, et al. Remote magnetic control of a wireless capsule endoscope in the esophagus is safe and feasible: results of a randomized, clinical trial in healthy volunteers. Gastrointest Endosc 2010;72(5):941-946

6 Keller J, Fibbe C, Volke F, et al. Inspection of the human stomach using remote-controlled capsule endoscopy: a feasibility study in healthy volunteers (with videos) Gastrointest Endosc 2011;73(1):22-28

7 Rahman I, Pioche M, Shim CS, et al. Magnetic-assisted capsule endoscopy in the upper GI tract by using a novel navigation system (with video) Gastrointest Endosc 2016;83(5):889895.e1

8 Rahman I, Kay M, Bryant T, et al. Optimizing the performance of magnetic-assisted capsule endoscopy of the upper GI tract using multiplanar CT modelling. Eur J Gastroenterol Hepatol 2015;27(4):460-466

9 Ching H-L, Hale MF, Sidhu R, Beg S, Ragunath K, McAlindon ME. Magnetically assisted capsule endoscopy in suspected acute upper GI bleeding versus esophagogastroduodenoscopy in detecting focal lesions. Gastrointest Endosc 2019;90(3):430-439

10 Rey J-F, Ogata H, Hosoe N, et al. Blinded nonrandomized comparative study of gastric examination with a magnetically guided capsule endoscope and standard videoendoscope. Gastrointest Endosc 2012;75(2):373-381

11 Denzer UW, Rösch T, Hoytat B, et al. Magnetically guided capsule versus conventional gastroscopy for upper abdominal complaints: a prospective blinded study. J Clin Gastroenterol 2015;49(2):101-107

12 Liao Z, Duan X-D, Xin L, et al. Feasibility and safety of magnetic-controlled capsule endoscopy system in examination of human stomach: a pilot study in healthy volunteers. J Interv Gastroenterol 2012;2(4):155-160
13 Ching H-L, Hale M, Sidhu R, McAlindon M. PTH-050 Robot magnet-controlled upper GI capsule endoscopy using the Ankon NaviCam ${ }^{\circledR}$ system: first reported experience outside china. Gut 2017;66(Suppl 2) :A230

14 Zou W-B, Hou X-H, Xin L, et al. Magnetic-controlled capsule endoscopy vs. gastroscopy for gastric diseases: a two-center self-controlled comparative trial. Endoscopy 2015;47(6):525-528

15 Liao Z, Hou X, Lin-Hu E-Q, et al. Accuracy of magnetically controlled capsule endoscopy, compared with conventional gastroscopy, in detection of gastric diseases. Clin Gastroenterol Hepatol 2016;14(9):1266-1273.e1

16 Jiang B, Qian Y-Y, Pan J, et al. Second-generation magnetically controlled capsule gastroscopy with improved image resolution and frame rate: a randomized controlled clinical trial (with video) Gastrointest Endosc 2020;91(6):1379-1387

17 Liao Z, Zou W, Li Z-S. Clinical application of magnetically controlled capsule gastroscopy in gastric disease diagnosis: recent advances. Sci China Life Sci 2018;61(11):1304-1309

18 Lien G-S, Wu M-S, Chen C-N, Liu C-W, Suk F-M. Feasibility and safety of a novel magnetic-assisted capsule endoscope system in a preliminary examination for upper gastrointestinal tract. Surg Endosc 2018;32(4):1937-1944

19 Chen Y-Z, Pan J, Luo Y-Y, et al. Detachable string magnetically controlled capsule endoscopy for complete viewing of the esophagus and stomach. Endoscopy 2019;51(4):360-364

20 Song J, Bai T, Zhang L, Xiang X-L, Xie X-P, Hou X-H. Better view by detachable string magnetically controlled capsule endoscopy for esophageal observation: a retrospective comparative study. Dis Esophagus 2020;33(4):doz104 [Internet]

21 Beg S, Card T, Warburton S, et al. Diagnosis of Barrett's esophagus and esophageal varices using a magnetically assisted capsule endoscopy system. Gastrointest Endosc 2020;91(4):773-781.e1

22 Hale MF, Drew K, Sidhu R, McAlindon ME. Does magnetically assisted capsule endoscopy improve small bowel capsule endoscopy completion rate? A randomised controlled trial. Endosc Int Open 2016;4(2):E215-E221

23 Kyoung-chul K, Cha J, Jeon D, Dong-il Dan C. A rotational micro biopsy device for the capsule endoscope. In IEEE; 2005 [cited 2020 Jun 20]. p. 1839-43. Available from: http://ieeexplore. ieee.org/document/1545441/. Accessed August 5, 2020

24 Valdastri P, Quaglia C, Susilo E, et al. Wireless therapeutic endoscopic capsule: in vivo experiment. Endoscopy 2008;40(12):979-982

25 Xia J, Xia T, Pan J, et al. Use of artificial intelligence for detection of gastric lesions by magnetically controlled capsule endoscopy. Gastrointest Endosc [Internet]. 2020 May [cited 2020 Jun 20]; Available from: https://linkinghub.elsevier.com/retrieve/ pii/S0016510720343625. Accessed August 5, 2020

26 Nam S-J, Lee HS, Lim YJ. Evaluation of gastric disease with capsule endoscopy. Clin Endosc 2018;51(4):323-328 